Radiologe $2011 \cdot 51: 784-790$

DOI 10.1007/s00117-011-2146-6

Online publiziert: 10. August 2011

(c) Springer-Verlag 2011

J.M. Lieb · S. Ulmer ${ }^{1}$. J. Kelm² $\cdot$ K. Shariat ${ }^{3}$. C. Stippich ${ }^{1}$ F.J. Ahlhelm ${ }^{1}$

${ }^{1}$ Abteilung für Diagnostische und Interventionelle Neuroradiologie,

Klinik für Radiologie und Nuklearmedizin, Universitätsspital Basel

${ }^{2}$ Chirurgisch-orthopädisches Zentrum Illingen, Illingen/Saarland

${ }^{3}$ Abteilung Stereotaktische Neurochirurgie,

Neurochirurgische Universitätsklinik Freiburg

\title{
Postoperative Befunde an der Wirbelsäule
}

\section{Röntgen, CT, Myelographie, MRT}

Degenerative Wirbelsäulenerkrankungen sind weit verbreitete Volkskrankheiten und aufgrund ihrer hohen Morbidität insbesondere von sozioökonomischer Bedeutung [1, 2]. Fortschritte in der bildgebenden Diagnostik und Weiterentwicklungen operativer Techniken haben dazu beigetragen, dass auch die Anzahl der Wirbelsäuleneingriffe in den letzten Jahren angestiegen ist [1]. Kommt es postoperativ kurz-, mittel- oder längerfristig zu neuen Beschwerden oder fehlt eine Beschwerdebesserung, werden zunehmend bildgebende Verfahren zeitnah zur Operation eingesetzt, deren Interpretation aufgrund operativer Veränderungen eine Herausforderung sein kann. Bei komplexen chirurgischen Eingriffen werden postoperative Kontrolluntersuchungen mithilfe der modernen Bildgebung heute routinemäßig durchgeführt. Dazu stehen Verfahren zur Verfügung, deren Indikation von komplexen Faktoren abhängt (initiale Pathologie, Operationsverfahren, Zugangsweg, individuelle Faktoren der Wundheilung, Intervall zwischen Operation und Symptombeginn). Bei unklaren bildgebenden Befunden kann neben der klinischen Symptomatik und den laborchemischen Befunden auch die Kombination mehrerer Verfahren hilfreich sein.
Konventionelle Röntgenbilder sind oft Standardverfahren nach spinalen Eingriffen mit Instrumentation zur Überprüfung der Implantatlage und zur Stellungskontrolle. Bei transpedikulärer Verschraubung reicht das konventionelle Röntgenbild zur Beurteilung der Intaktheit angrenzender neuraler Strukturen manchmal nicht aus und ist der Schnittbilddiagnostik (CT und MRT) unterlegen. Aufgrund der hohen Verfügbarkeit ist die CT immer noch die häufigste Schnittbilddiagnostik nach Wirbelsäuleneingriffen und ermöglicht dank moderner Technik (Mehrzeilenspiraltechnik) orthogonale Reformationen in allen Ebenen sowie 3-D-Darstellungen. Metallaufhärtungsartefakte schränken die Beurteilbarkeit im CT in der Implantatregion jedoch häufig ein, zudem verwendet diese Untersuchung ionisierende Strahlung.

Die Myelographie bzw. ergänzend das Myelo-CT erlaubt nach invasiver, intrathekaler Kontrastmittel(KM)-Injektion die genaue Beurteilung der Konturen des Myelons, der Kaudafasern und der Wurzeltaschen sowie zusätzlich der knöchernen Strukturen. Des Weiteren ist mit dieser Untersuchung eine dynamische Beurteilung hinsichtlich funktioneller Instabilitäten wie z. B. Kompression des Myelons und der Nervenwurzeln bei diskoligamentären Insuffizienzen möglich. Mittels MRT lassen sich aufgrund des guten Weichteilkontrasts und der Möglichkeit des Nachweises eines Knochenödems sowohl knöcherne als auch Weichteilstrukturen im Detail beurteilen. Postoperativ stellt sie eine gute Möglichkeit dar, prozedural bedingte oder postoperativ im Verlauf aufgetretene Pathologien oder peri-/ postoperative Komplikationen mit hoher Sensitivität und Spezifität zu erfassen, wie dieser Beitrag zeigen soll. Manche (Weichteil-)Pathologien lassen sich generell sowohl prä- als auch postoperativ nur mit der MRT, wie z. B. die Syringomyelie (• Abb. 1) darstellen, da diese im Röntgenbild und auch CT oder Myelo-CT nicht zu beurteilen ist.

Die Implantat- bzw. auch durch Schraubenabrieb bedingten Suszeptibilitätsartefakte im MRT hängen sowohl von der gewählten Sequenztechnik als auch von der maximalen Magnetflussdichte ab. Bei 1,5 Tesla eignen sich (Turbo-) Spinechosequenzen deutlich besser für die postoperative Bildgebung aufgrund der geringeren Suszeptibilitätsartefaktanfälligkeit im Vergleich zu Gradientenecho(GRE)-Sequenzen (• Abb. 2). Bei 3,o Tesla dagegen zeigen GRE-Sequenzen eine geringere Suszeptibilitätsartefaktanfälligkeit. Während ferromagnetische Materialien deutlich höhere Suszeptibilitätsartefakte aufweisen (z. B. bei eisenhaltigen Implantaten), sind diese bei modernen, diamagnetischen Materialien/Legierungen (Titan, Aluminium, Vanadium) weniger ausgeprägt [1]. Insgesamt eignen sich fettsupprimierte Sequenzen weniger gut zur Datenakquisition bei einliegen- 
dem Fremdmaterial, da die Fettsuppression durch die Fremdmaterialien gestört wird und dies zu zusätzlichen Artefakten führt. Eine alternative Möglichkeit stellt die Anfertigung nicht fettgesättigter nativer und kontrastverstärkter T1w-Sequenzen mit anschließender Subtraktionstechnik dar (sofern keine Bewegungsartefakte vorliegen).

\section{Unmittelbar postoperative oder frühzeitige Komplikationen}

Hierzu zählen:

- iatrogene Verletzung knöcherner oder neuraler Strukturen,

- Implantatfehllage,

- postoperatives Hämatom,

- entzündliche Veränderungen (septisch z. B. Spondylodiszitis) oder aseptisch (z. B. Arachnoiditis) und - Rezidivprolaps.

\section{Postoperatives Hämatom}

Kleinere Hämatome im chirurgischen $\mathrm{Zu}$ gangsweg werden nach Wirbelsäuleneingriffen häufig beobachtet. Klinisch relevante, intraspinale Hämatome/epidurale Blutungen mit neuer neurologischer Symptomatik sind seltener (laut einer systematischen Metaanalyse von Glotzbecker et al. [7] beträgt die Inzidenz $0-1 \%$ ), gehen aber mit einer hohen potenziellen Morbidität einher und bedürfen häufig einer umgehenden notfallmäßigen Revision. Die Größe eines Hämatoms korreliert dabei nicht zwingend mit dem Ausmaß der Symptomatik, sodass von einer standardisierten postoperativen Bildgebung abgeraten wird; federführend ist die klinische Symptomatik. Die Darstellung des postoperativen Hämatoms gelingt am besten mittels MRT, wobei nach einer Instrumentation Suszeptibilitätsartefakte die Beurteilbarkeit erschweren können.

\section{Rezidivprolaps}

Die MRT ist die Methode der Wahl für die Differenzierung zwischen einem Rezidivprolaps und Narbengewebe [3, 4, 9]. Die MR-tomographische Unterscheidung zwischen dem Rezidivprolaps und typischen, narbigen postoperativen Veränderungen ist häufig schwierig und ge-

\section{Radiologe 2011 · 51:784-790 DOI 10.1007/s00117-011-2146-6}

(c) Springer-Verlag 2011

\section{J.M. Lieb · S. Ulmer · J. Kelm · K. Shariat · C. Stippich · F.J. Ahlhelm Postoperative Befunde an der Wirbelsäule}

\section{Zusammenfassung}

Die postoperative Bildgebung wird klassischerweise herangezogen zur Dokumentation der korrekten Implantatlage oder um Komplikationen auszuschließen, wenn der Patient postoperativ weiterhin Beschwerden angibt. In Abhängigkeit von der Fragestellung können verschiedene Modalitäten verwendet werden - alle mit Vor- und Nachteilen.

Die konventionelle Röntgenuntersuchung wird zur Dokumentation der Implantatlage, Beurteilung der Stabilität oder im Follow-up zur Frage der Instabilität oder einer Implantatfraktur verwendet, wogegen Weichteilveränderungen nicht komplett beurteilt werden können. Neben diesen Indikationen wird eine Bildgebung bei persistierenden Beschwerden (meist Schmerzen) des Patienten veranlasst.
Residuelles oder rezidiviertes Bandscheibengewebe, ein Hämatom oder eine Entzündung können am besten mit der MRT beurteilt werden. Die MRT sollte unmittelbar postoperativ durchgeführt werden, um eine physiologische Granulation im Zugangsgebiet von entzündlichen Veränderungen unterscheiden zu können. Oft kann die Bildgebung allein dies nicht unterscheiden, daher ist die Bildgebung nur ein weiteres Puzzelstück. Die Computertomographie ist die Modalität der Wahl zur Beurteilung von Knochen und eine Ergänzung bei neuen Verfahren wie der bildgestützten Kypho- oder Vertebroplastie.

\section{Schlüsselwörter}

Postoperativ · Wirbelsäule · Chirurgie · Bildgebung · Artefakte

\section{Postoperative findings in the spinal column}

\section{Abstract}

Postoperative imaging after spinal surgery is usually performed to document the correct positioning of implants or to rule out complications if patients still suffer from pain after surgery. Depending on the question various imaging modalities can be used all of which have benefits and limitations.

Conventional $\mathrm{X}$-ray is used for the documentation of the correct positioning of spinal implants, stability (olisthesis) and during follow-up to rule out fractures or instability of the implants, whereas soft tissue changes cannot be completely assessed. Besides these indications, imaging is usually performed because of ongoing symptoms (pain for the most part) of the patients. Soft tissue changes including persistent or recurrent herniated disc tissue, hematoma or infection can best be depicted using magnetic resonance imaging (MRI) which should be performed within the immediate postoperative period to be able to distinguish physiological development of scar tissue from inflammatory changes in the area of the surgical approach. Often imaging alone cannot differentiate between these and imaging can therefore only be considered as an adjunct. Computed tomography is the modality of choice for the evaluation of bony structures and an adjunct of new therapies such as image-guided application of cement for kyphoplasty or vertebroplasty.

Keywords Postoperative $\cdot$ Spine $\cdot$ Surgery $\cdot$ Imaging . Artifacts 

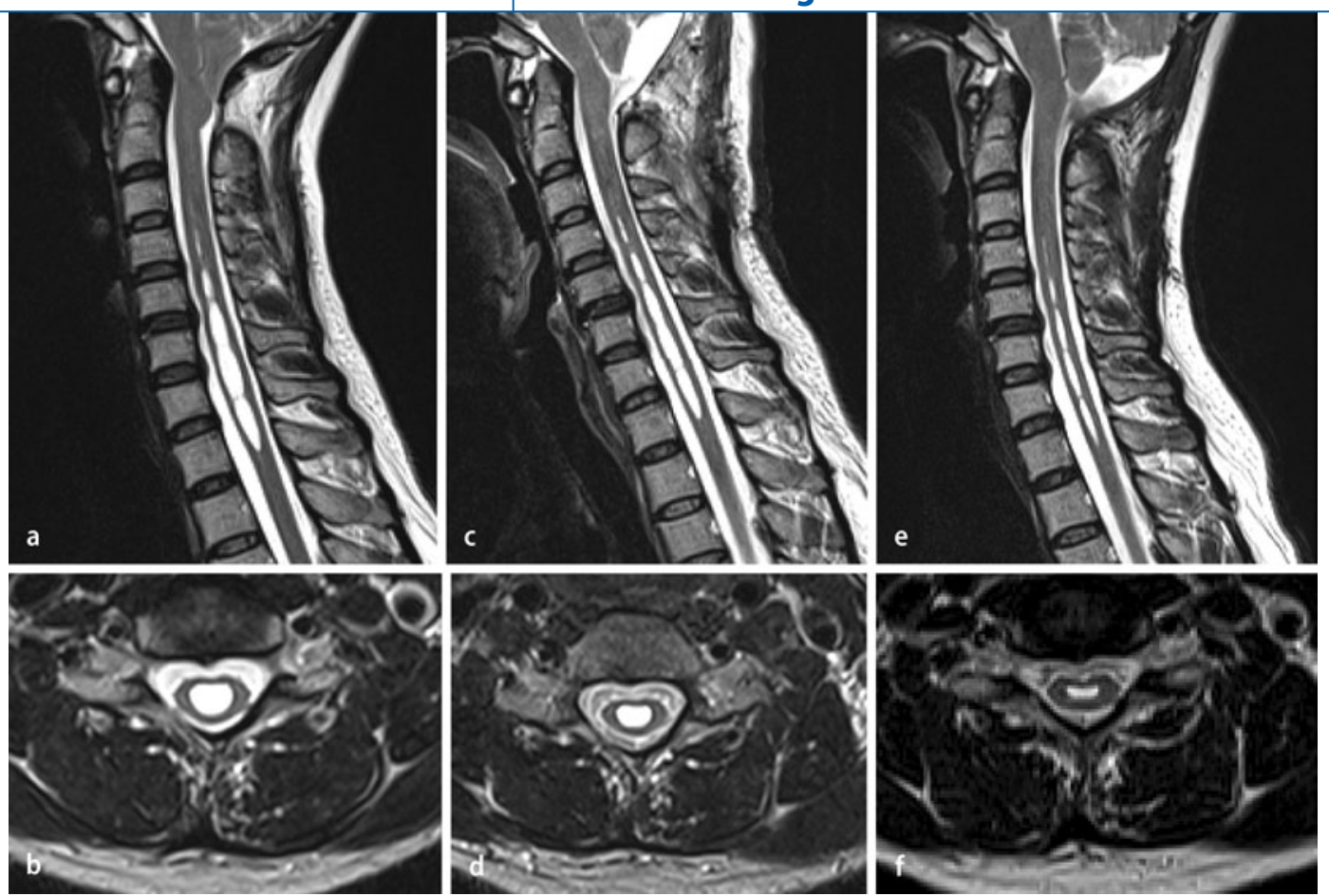

Abb. $1 \varangle$ MRT der Halswirbelsäule, T2w-Sequenzen sagittal $(\mathbf{a}, \mathbf{c}, \mathbf{e})$ und transversal $(\mathbf{b}, \mathbf{d}, \mathbf{f})$. Patient mit Chiari-II-Malformation und einer Syringohydromyelie. a, b Präoperative MRTUntersuchung vor subokzipitaler Dekompression. c-f Postoperativer Verlauf mit Regredienz der Syrinx. Die Syringomyelie zählt zu den Krankheitsbildern, die primär und auch im Verlauf mittels MRT untersucht werden sollten
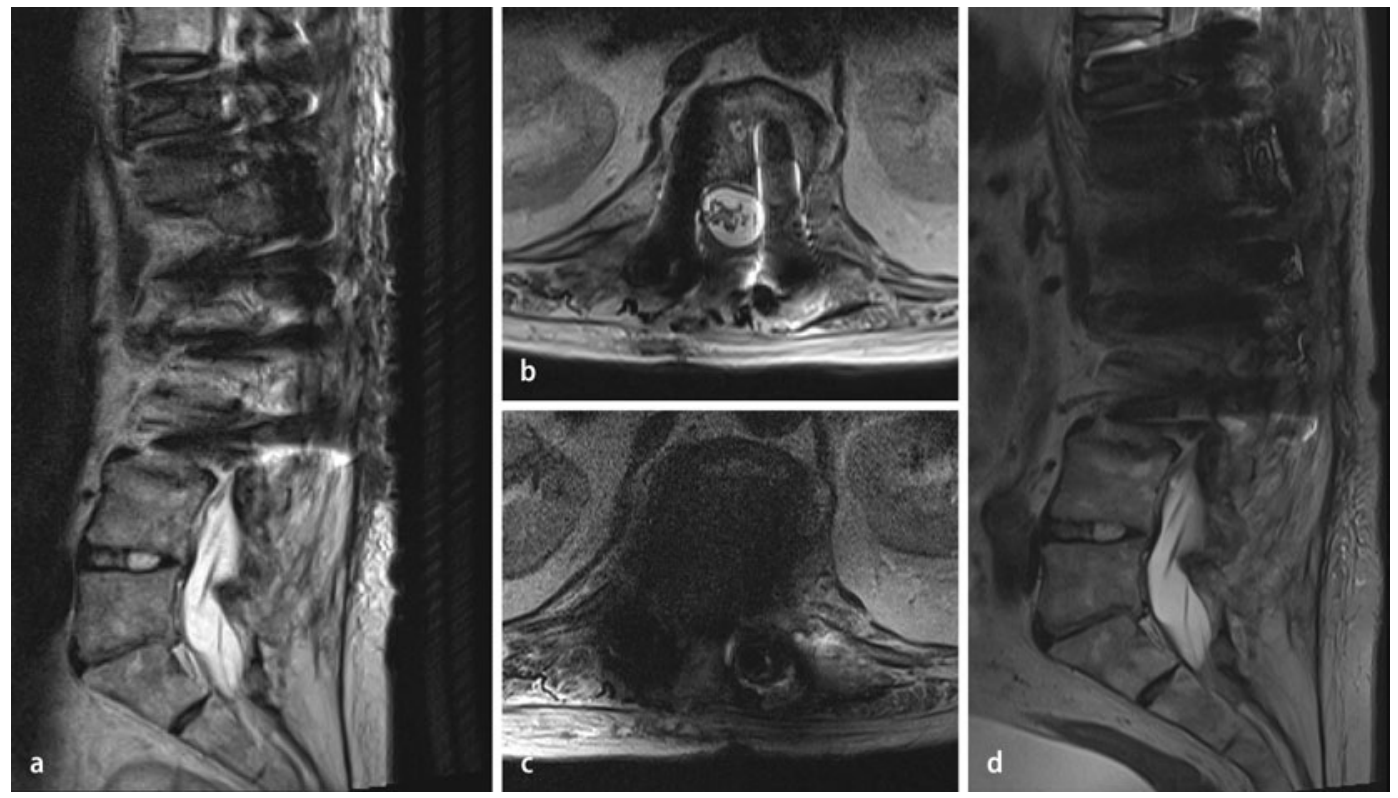

Abb. $2 \varangle$ MRT der Lendenwirbelsäule nach dorsaler, mehrsegmentaler Spondylodese. T2w-TSE-Sequenz sagittal (a) und transversal (b) bei 1,5 T mit deutlich weniger Suszeptibilitätsartefakten im Vergleich zu 3,0 Tesla (c und d) beim selben Patienten. Anders verhält es sich bei Gradientenechosequenzen, diese zeigen bei 3,0 Tesla deutlich weniger Artefakte (kein Bildbeispiel). TSE Turbospinecho

lingt meist nur durch eine zusätzliche Kontrastmittelgabe (• Abb. 3). Während Bandscheibengewebe avaskulär ist und nicht KM aufnehmend, zeigt Narbengewebe ein deutliches Enhancement. Innerhalb von 3 bis 6 Monaten kann es postoperativ zur überschießenden Narbenbildung aber auch Schrumpfung narbiger Prozesse kommen (s. Failed-back-surgery-Syndrom). Bei unmittelbar postoperativ persistierenden bzw. zeitnah wieder auftretenden Beschwerden wird ein MRT empfohlen, um zwischen Rezidivvorfall,
Narbengewebe mit oder ohne begleitender Flüssigkeitsansammlung und Hämatom unterscheiden zu können.

\section{Implantatfehllage/-versagen}

Klassisch erfolgt die intraoperative Dokumentation der Implantate mittels Durchleuchtung (C-Bogen). Wenn verfügbar, können mittels intraoperativer CT-Bildgebung eine Implantatfehllage (z. B. transpedikuläre Schraubenfehllage) frühzeitig festgestellt werden [1]. Besteht postopera- tiv nach Röntgenaufnahmen oder bei entsprechender Klinik der Verdacht auf eine Implantatfehllage, wird dies meist mittels CT (Mehrzeilenspiral-CT und multiplanare Reformationen) weiter abgeklärt.

Je nach Implantatmaterial und Gerätetechnologie ist auch hier mit unterschiedlich ausgeprägten Aufhärtungsartefakten $\mathrm{zu}$ rechnen. Titanlegierungen verursachen die wenigsten, Edelstahl die stärksten Artefakte. Aufhärtungsartefakte können z. T. durch neue Gerätetechnologie reduziert werden, wie z. B. Multidetec- 

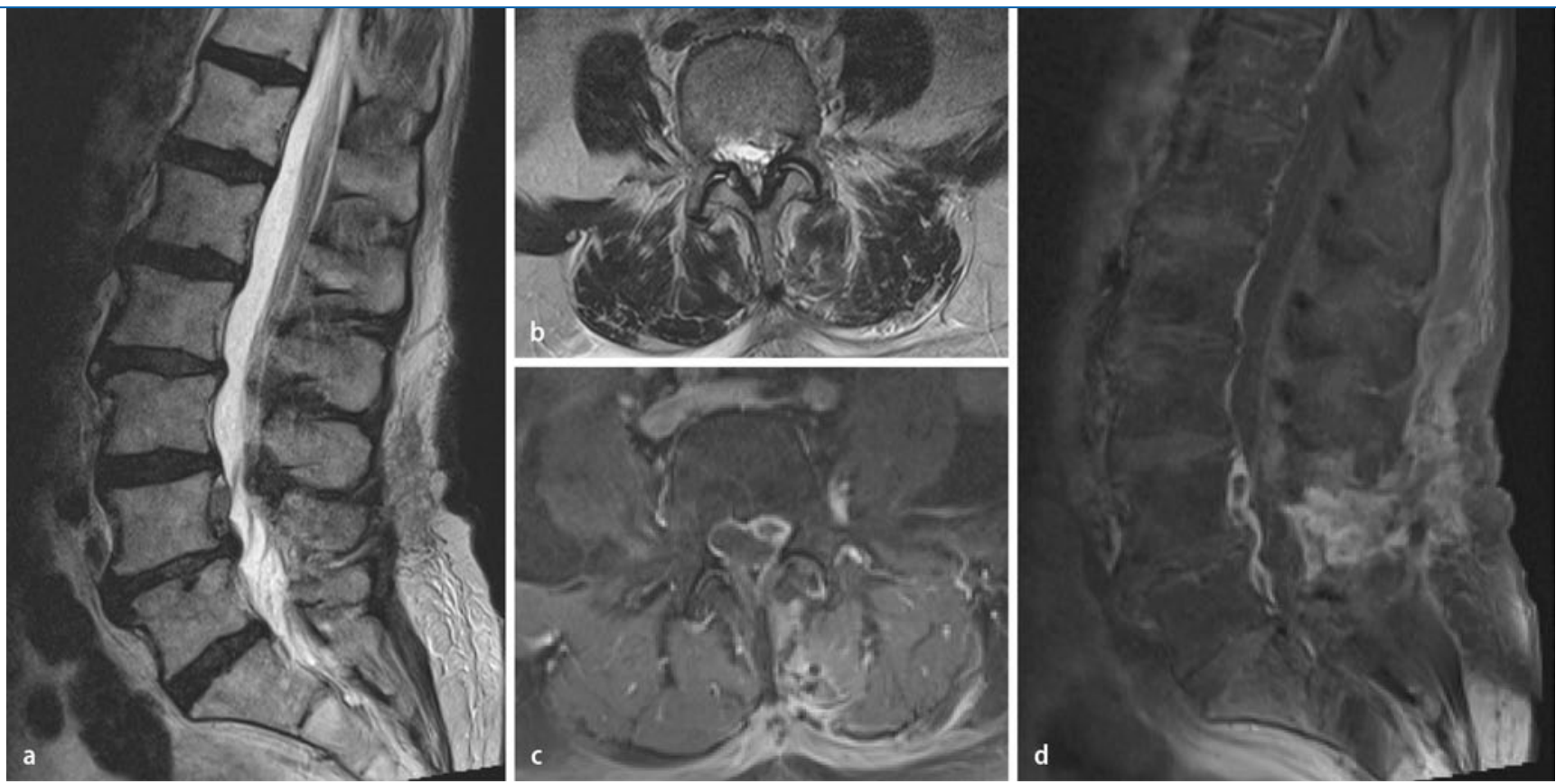

Abb. $3 \triangle$ MRT der Lendenwirbelsäule. In sagittaler (a) und transversaler (b) T2w-Untersuchungstechnik. Nachweis typischer postoperativer Veränderungen sowie eines Weichteilplus rezessal links. Nach Kontrastmittelgabe erkennt man in axialer (c) und sagittaler (d) T1w-Untersuchungstechnik das avaskuläre, nicht KM aufnehmende Bandscheibenprolapsrezidiv mediolateral links mit rezessaler Verlegung und Kompression angrenzender neuraler Strukturen. KM Kontrastmittel

tor-CTs, durch Erhöhung der Spannung und des Röhrenstroms (mit Erhöhung der Strahlenexposition), Anpassung der Kollimation und Schichtdicke sowie durch Postprocessing, wie z. B. Erhöhung der Schichtdicke in der Rekonstruktion, Glättungsfilter (Kernel) und Ausdehnung der Hounsfield-Skala von einem Standardmaximum von 4000 auf $40.000 \mathrm{HU}$ [10].

Weitere postoperative Befunde nach Instrumentation, die meist erst im längerfristigen Verlauf auftreten, sind Implantatlockerung oder -bruch (Implantatversagen). Bildmorphologisch werden bei einem Bruch des Implantats neben einer Aufhellungslinie auch eine Dislokation von Osteosynthesematerial gefunden sowie ein radioluzenter Saum zwischen Implantatoberfläche und Knochenstruktur bei Lockerung. Eine Implantatlockerung kann zu erneuter Bewegung/Instabilität im versteiften Wirbelsäulenabschnitt und dadurch zu Schmerzen führen.

\section{Infektion (Diszitis, Spondylodiszitis)}

Die postoperative Diszitis tritt mit einer Häufigkeit von etwa 0,2 bis zu 5,6\% auf, dabei wird die septische und die aseptische Form unterschieden $[8,11]$. Es ist wichtig, die septische Form zu identifi- zieren, da diese zumindest eine antibiotische Therapie bzw. auch eine operative Sanierung erfordert. Häufigster ursächlicher Erreger ist Staphylococcus aureus, der oft mittels bildgesteuerter Punktion und Probeentnahme für eine gezielte Antibiose nachgewiesen werden kann. Klinisch bleibt die peri-/postoperative Infektion anfänglich häufig unspezifisch mit Schmerzen und steigenden Entzündungsparametern. Für das klinische Monitoring eignet sich das C-reaktive Protein (CRP) dank der hohen Sensitivität. Bildmorphologisch ist die MRT die Methode der Wahl. Gefunden werden ein Kontrastmittelenhancement der Disci und der angrenzenden Wirbelkörper sowie eine Signalsteigerung der betroffenen Bandscheibe in T2-Wichtung und Signalabsenkung der Wirbelkörper in Tiw aufgrund der Flüssigkeitseinlagerung [5]. Zudem findet sich häufig in der angrenzenden paravertebralen Muskulatur eine Signalsteigerung in T2-gewichteter Technik. Eine weitere Komplikation stellt die Abszedierung dar, die bildmorphologisch als Flüssigkeitskollektion mit randständigem Enhancement und Umgebungsödem imponiert. Weitere Informationen zur Spondylodiszitis und deren Komplikationen können dem entsprechenden Leitartikel des Themenhefts entnommen werden.

\section{Abszedierung}

Die Abszedierung als postinfektiöse Komplikation kann in unterschiedlichen Kompartimenten stattfinden, am häufigsten paravertebral oder epidural. Bildmorphologisch imponiert die Abszedierung als Flüssigkeitskollektion mit Randenhancement und Umgebungsreaktion (Bildbeispiel s. Artikel „Entzündliche Erkrankungen der Wirbelsäule und des Myelons" in diesem Heft.)

\section{Peridurale Fibrose}

Zu einer periduralen Fibrose nach wirbelsäulenchirurgischen Eingriffen kommt es in der Regel generell als normale postoperative Reaktion. Diese fibrotische Reaktion und die Narbenbildung können in unterschiedlichem Maß auftreten und führen zu einer Infiltration des epiduralen/perineuralen Fettgewebes auf Operationshöhe. Nach Kontrastmittelgabe zeigt dieses Narbengewebe bereits innerhalb von 3 Tagen postoperativ ein kräftiges Enhancement und kann u. U. von einer Entzündung bildmorphologisch kaum mehr 

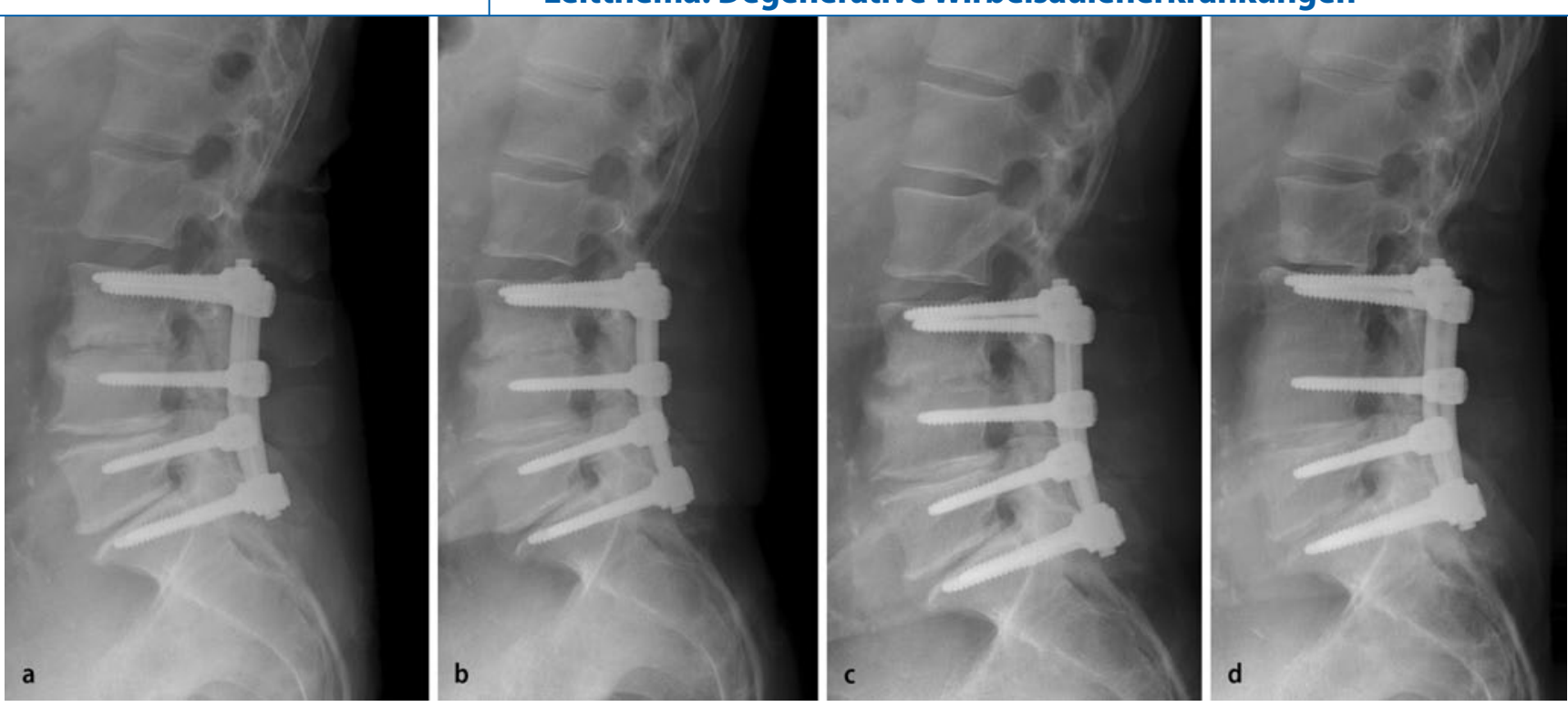

Abb. $4 \Delta$ Röntgen der Lendenwirbelsäule im lateralen Strahlengang. Postoperativer Befund nach Spondylodese LWK3 bis SWK1 (a), nach 3 Monaten (b), nach 6 Monaten (c), nach 3 Jahren (d) mit Entwicklung einer Anschlussdegeneration im Bewegungssegment LWK2/3 unmittelbar kranial der Spondylodese sowie zunehmender ossärer Fusion der stabilisierten Segmente
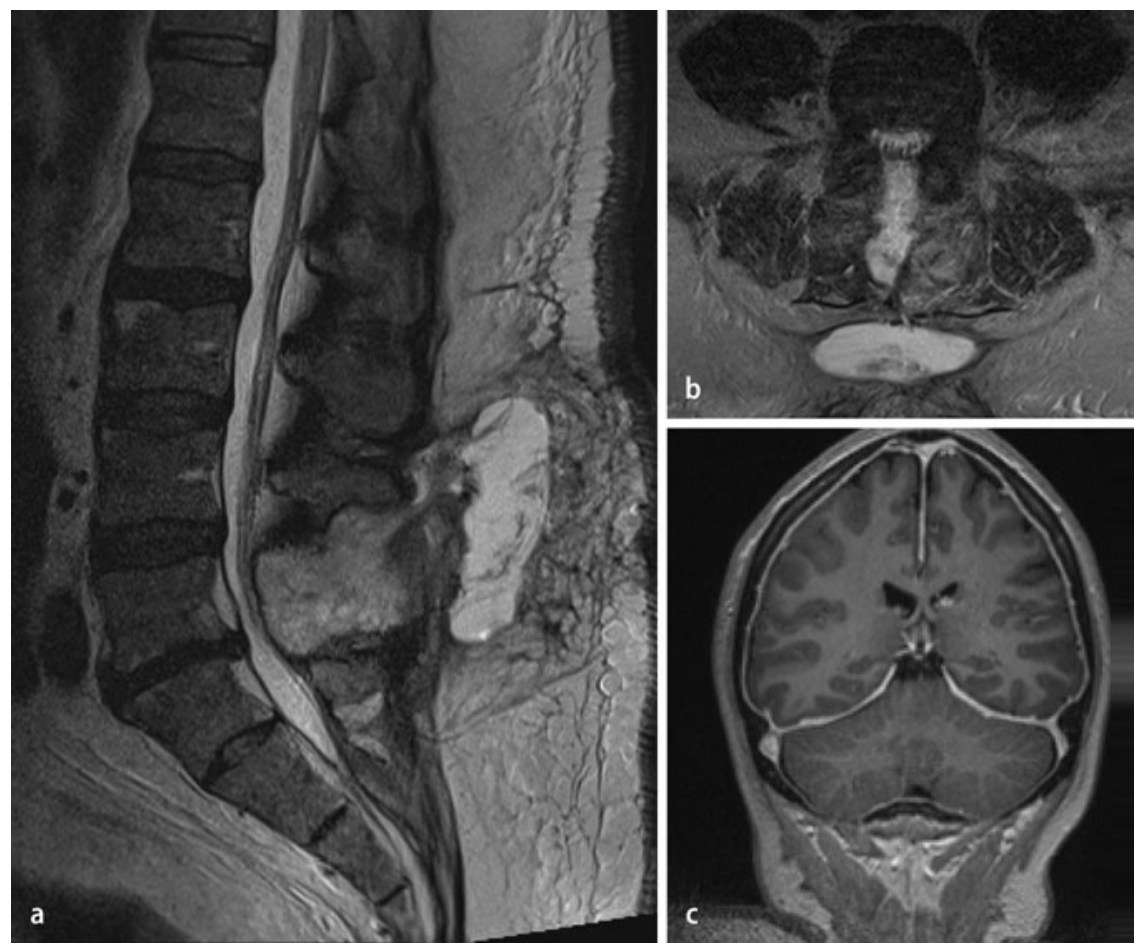

unterschieden werden. Hier sind häufig nur die Klinik und das Zeitfenster zwischen Operation und Bildgebung richtungsweisend. Über die Zeit zeigt dieses Narbengewebe eine Schrumpfungstendenz und kann dadurch zu Scher-/Zugkräften auf den Duralsack und die Wurzeln führen mit entsprechender klinischer Symptomatik. Die peridurale Fibrose kann laut Gil u. Frymoyer [6] für bis zu $5 \%$ der postoperativ schlechten Verläufe verantwortlich gemacht werden.

\section{Anschlussdegeneration}

Die Fusion von Bewegungssegmenten an der Wirbelsäule kann in den angrenzenden Bewegungssegmenten zu einer zusätzlichen Belastung mit beschleunigter Degeneration betreffender Bandscheibenfächer und Facettengelenke führen. Bildmorphologisch finden sich die typischen Zeichen der Degeneration mit höhengeminderten Bandscheibenfächern und

Abb. $5 \Delta$ Spinale MRT-Bildgebung der Lendenwirbelsäule in T2W-TSE-Technik in sagittaler (a) und transversaler (b) Akquisition. Nach operativer Dekompression Nachweis einer liquorisointensen, abgekapselten Flüssigkeitskollektion im Sinne einer Pseudomeningozele im Resektionsareal mit Kontakt zum Thekalsack sowie Fistelung und Depotbildung nach subkutan. Die KM-verstärkte, koronar akquirierte T1w-Untersuchung des Neurokraniums (c) zeigt eine generalisiert verdickte, KM-affine Pachymeninx als Zeichen eines Liquorunterdrucks infolge des Liquorlecks. Die kranielle Bildgebung wurde bei klinisch orthostatischer Kopfschmerzsymptomatik und Verdacht auf ein Liquorunterdrucksyndrom ergänzend angefertigt. TSE Turbospinecho, KM Kontrastmittel Gaseinschlussphänomen, Bandscheibenprotrusion und -prolaps, Spondylarthrosen, osteochondrotischen Veränderungen und gegebenenfalls Malalignement (- Abb. 4). Klinisch zeigen die Patienten typischerweise Rückenbeschwerden mit pseudoradikulärer Symptomatik sowie bei zusätzlicher foraminaler Einengung radikuläre Beschwerden wie $\mathrm{z}$. B. eine Brachialgie oder Ischialgie. Weitere 
Informationen zur Wirbelsäulendegeneration können dem entsprechenden Leitartikel des Themenhefts entnommen werden.

\section{Instabilität}

Mit zunehmender knöcherner Dekompression (Teilhemilaminektomie $<$ Hemilaminektomie <Laminektomie) kann es insbesondere bei mehrsegmentalen Eingriffen im Verlauf zu einer Wirbelsäuleninstabilität und Olisthesis kommen. Diese nimmt typischerweise bei Bewegung und auch über die Zeit zu. Die Diagnose erfolgt häufig schon mit der konventionellen Röntgenuntersuchung einschließlich Funktionsaufnahmen.

\section{Pseudomeningozele und Liquorunterdrucksyndrom}

$\mathrm{Zu}$ einer Pseudomeningozelenbildung nach spinalem Eingriff kommt es in etwa 0,19-2\% der Fälle, meist dorsal des Thekalsacks auf der Seite der Laminektomie. Bei der Pseudomeningozele handelt es sich um ein durales Leck mit Ansammlung oder Austritt von Liquor mit Ausbildung einer Pseudokapsel. Bildmorphologisch stellt sich eine liquorisointense zystische Formation im Operations-/Zugangsgebiet mit Assoziation zum Thekalsack und ggf. Fistelbildung bis nach subkutan dar (• Abb. 5a). Klinisch bleibt die Pseudomeningozele häufig asymptomatisch, kann in Abhängigkeit der Ausdehnung palpabel werden oder bei assoziiertem Liquorunterdruck zu Kopfschmerzen führen. Typisch für das Liquorunterdrucksyndrom sind lageabhängige/orthostatische Kopfschmerzen mit Zunahme bei Aufrichtung der Körperachse. Bildmorphologisch typisch findet sich in der kraniellen Bildgebung ein prominentes meningeales Enhancement (• Abb. 5b ). Bei ausgeprägtem Unterdruck kann es zusätzlich zu einer intrakraniellen Hygrombildung kommen. In $75 \%$ der Fälle erfolgt eine spontane Besserung innerhalb von 3 Monaten mit Regredienz der Pseudomeningozele und des Liquorunterdrucksyndroms.
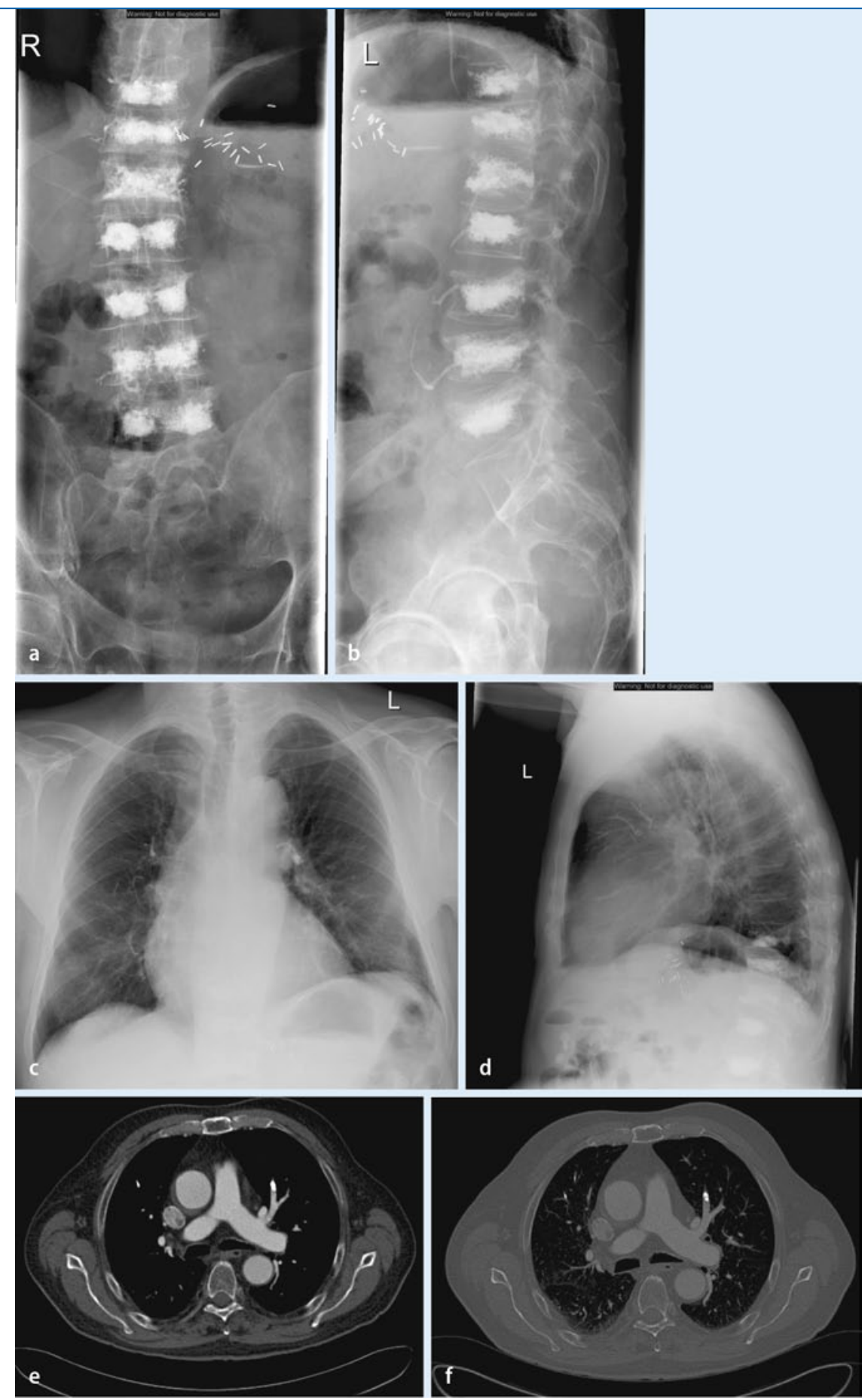

Abb. $6 \Delta$ Röntgen der Lendenwirbelsäule im p.-a.- (a) und lateralen (b) Strahlengang nach mehrsegmentaler Vertebroplastie; Nachweis von Zementaustritt in lumbale Venen. In der Röntgenuntersuchung des Thorax in p.-a.- (c) und lateraler (d) Projektion lassen sich als seltene Komplikation Zementemboli in den Lungenarterien nachweisen. Auch in der kontrastmittelverstärkten CT des Thorax lassen sich im Knochen- (e) und Weichteilfenster (f) röntgendichte Embolien in der Lungenstrombahn feststellen

\section{Arachnoiditis/Arachnopathie}

Im Zusammenhang mit einer entzündlichen Erkrankung der Wirbelsäule, wirbelsäulenchirurgischen Eingriffen oder intrathekaler Injektion von Medikamen- ten oder Kontrastmitteln kann es begleitend zu einer Arachnoiditis/Arachnopathie (auch Arachnoiditis adhaesiva, Menigitits serosa circumscripta genannt) kommen [12]. Hierzu s. auch den Abschnitt Arachnoiditis/Arachnopathie im Kapitel 
„Entzündliche Erkrankungen der Wirbelsäule und des Myelons" mit entsprechenden Bildbeispielen in diesem Heft.

\section{Kypho-/Vertebroplastie und extrakorporaler Zement}

Als Komplikation bei der Vertebroplastie, die als minimalinvasives Stabilisierungsverfahren für osteoporotische oder pathologische Wirbelkörperfrakturen zunehmend an klinischer Bedeutung gewinnt, kann es nach intrakorporaler Polymethylmetaacrylat(PMMA)-Applikation auch gelegentlich zu Austritten dieses „Knochenzements“ in die lumbalen intraund paraspinalen Venenplexus kommen. Als Folge können eine spinale Raumforderung oder bei Aushärtung des PMMA eine thermische Schädigung neuronaler Strukturen und sogar Zementembolien in der Lunge resultieren (• Abb. 6). Um dies zu verhindern, wurde die Applikation unter CT-Kontrolle (CT-Fluoroskopie) empfohlen, die zu jedem Zeitpunkt eine Bildkontrolle der Zementapplikation ermöglicht und der klassischen Durchleuchtung deutlich überlegen ist. Durch die Entwicklung neuerer Zementmischungen bzw. -zusammensetzungen wird die Methodik derzeit schon deutlich verbessert und bietet eine höhere therapeutische Sicherheit und Reduktion von Komplikationen.

\section{Failed-back-surgery-Syndrom (FBSS)}

Zum Failed-back-surgery-Syndrom werden eine klinische Symptomatik oder ein Symptomkomplex zusammengefasst, der sich pathogenetisch aus unterschiedlichen Entitäten zusammensetzt, die u. a. auch prozedural bedingt sind. Häufig erleben Patienten nach Wirbelsäuleneingriffen keine oder nur eine geringe Verbesserung oder sogar eine klinische Verschlechterung. Laut Schofferman et al. [13] sind die häufigsten Ursachen des FBSS die foraminäre Stenose (25-29\%), schmerzhafte Bandscheibe („painful disc“, 2022\%), Rezidivprolaps (7-12\%) oder seltene Komplikationen wie z. B. Pseudoarthrosen, neuropathische Schmerzen, Facettenarthrosen, Instabilitäten $[1,13]$. Hinzu kommt die typische Ausbildung von Nar- bengewebe (epidurale Fibrose) postoperativ, die - wie bereits erwähnt - ebenfalls für bestehende Beschwerden verantwortlich sein kann. Bei der foraminären Stenose handelt es sich um eine konsekutive Einengung des Neuroforamens bedingt durch die Höhenminderung nach Diskektomie.

\section{Fazit für die Praxis}

- Mit der modernen Bildgebung lassen sich postoperative Komplikationen nach wirbelsäulenchirurgischen Eingriffen wie eine periprozedurale Blutung oder ein frühes Bandscheibenprolapsrezidiv nachweisen.

- Manchmal ist der Einsatz verschiedener Verfahren wie z. B. MRT und Myelo-CT notwendig.

- Die Wahl des bildgebenden Verfahrens hängt $u$. a. von der Lokalisation und der Art des Eingriffs ab.

- Die MRT ermöglicht in vielen Fällen die adäquate Diagnose, kann jedoch insbesondere durch Metallimplantate oder allein durch Metallabrieb z. B. beim Fräsen durch Artefakte gestört werden.

- Neben der Bildgebung sind auch die klinische Symptomatik und laborchemische Untersuchungen für die Diagnose entscheidend.

\section{Korrespondenzadresse \\ PD Dr. F.J. Ahlhelm}

Abteilung für Diagnostische und Interventionelle Neuroradiologie, Klinik für Radiologie und Nuklearmedizin, Universitätsspital Basel,

Petersgraben 4, CH-4031 Basel, Schweiz ahlhelmf@uhbs.ch

Interessenkonflikt. Der korrespondierende Autor gibt an, dass kein Interessenkonflikt besteht.

\section{Literatur}

1. Ahlhelm F, Reith W, Naumann N et al (2006) Postoperatives Syndrom nach Wirbelsäuleneingriffen. Radiologe 46:486-494

2. Borenstein DG (1997) Epidemiology, etiology, diagnostic evaluation, and treatment of low back pain. Curr Opin Rheumatol 9:144-150

3. Bundschuh CV, Stein L, Slusser JH et al (1990) Distinguishing between scar and recurrent disc in postoperative patients: value of contrast-enhanced CT and MR imaging. AJNR Am J Neuroradiol 11:949-958
4. Bundschuh CV, Modic MT, Ross JS et al (1988) Epidural fibrosis and recurrent disk herniation in the lumbar spine: MR imaging assessment. AJR Am J Roentgenol 150(4):923-932

5. Dagirmanjian A, Schils J, McHenry M, Modic MT (1996) MR imaging of vertebral osteomyelitis revisited. AJR Am J Roentgenol 167:1539-1543

6. Gil K, Frymoyer JW (1991) The management of treatment failure after decompressive surgery. In: Frymoyer JW (ed) The adult spine: principles and practice. Lippincott-Raven, New York, pp 849-870

7. Glotzbecker MP, Bono CM, Wood KB, Harris MB (2010) Postoperative spinal epidural hematoma. Spine 35(10):413-420

8. Grane P, Josephsson A, Seferlis A, Tullberg T (1998) Septic and aseptic post-operative discitis in the lumbar spine - evaluation by MR imaging. Acta Radiol 39:108-115

9. Katzman GL (2004) Post-operative infection. In: Ross JS et al (eds) Diagnostic imaging spine VII (1). Amirsys Friesens, Altona, Manitoba, Canada, pp 38-42

10. Lee MJ, Sungjun K, Sung-Ah L et al (2007) Overcoming artifacts from metallic orthopedic implants at high-field strength MR imaging and multidetector CT. Radiographics 27:791-803

11. Lindholm TS, Pylkkanen P (1982) Diskitis following removal of intervertebral disk. Spine 7:618-622

12. Ross JS, Masaryk TJ, Modic MT et al (1987) MR imaging of lumbar arachnoiditis. AJR Am J Roentgenol 149(5):1025-1032

13. Schofferman J, Reynolds J, Herzog R et al (2003) Failed back surgery: etiology and diagnostic evaluation. Spine J 3(5):400-403 